\title{
Esthétique de la subjectivité dans \\ Je t'offrirai une gazelle de Malek Haddad
}

\author{
Messaoudi Samir \\ Université de Jijel
}

\section{Résumé}

Nous avons essayé, dans cet article portant sur la subjectivité dans Je t'offrirai une gazelle de Malek Haddad, de démontrer que le roman du poète est à la fois un texte poétique, traversé par la subjectivité et ancré dans son contexte (période coloniale). Contrairement au procès fait par l'essayiste Mostefa Lacheraf, qui a qualifié la production littéraire haddadienne de "croûte poétique», nous avons défendu, en partant de l'analyse de Je t'offrirai une gazelle, l'idée d'une œuvre poétique et engagée. Une œuvre qui refuse la colonisation, et qui prône l'indépendance du pays d'origine.

Mots-clés : Roman, Esthétique, Subjectivité, Colonisation, Écriture. 


\section{Introduction}

Publié en 1959 aux éditions Julliard, Je t'offrirai une gazelle de Malek Haddad raconte l'histoire d'un écrivain algérien en exil à Paris. Un jour il décide de publier un récit sans nom d'auteur. Le texte en question rapporte - deux récits enchâssés - un double échec : sentimental et littéraire ; l'amour impossible entre Yaminata et Moulay; et l'impossibilité de publier un manuscrit. Une fiction, celle de la publication du roman, qui se déroule dans un contexte particulier, celui de la période coloniale.

À la lecture du roman, notre attention est retenue par son esthétique : un récit poétique travaillé par la technique de mise en abyme. Écrit à la première personne du singulier - surtout dans les premières pages du récit -, le texte est traversé par un « je » très affiché. Avec les aléas du monde de l'édition auxquels est confronté le personnage-narrateur du récit, l'auteur semble rapporter, par le biais de la fiction, son expérience d'écrivain « francophone » durant la période coloniale.

En écrivant dans la langue de l'Autre, lequel représentant durant la période coloniale le colonialisme français, M. Haddad n'a pas caché son malaise d'écrire dans un idiome « étranger ». Ce « saut dans la gueule du loup », pour paraphraser le mot de Kateb Yacine, qui s'apparente à un déchirement linguistique, et partant à un exil intérieur, est exprimé à travers des procédés réflexifs. Nous reviendrons sur cette question dans notre analyse.

À travers la littérarité du texte - recours à l'esthétique de mise en abyme -, dans un contexte marqué par la guerre d'Algérie où des écrivains algériens sont « contraints » de défendre la cause de leur patrie, nous nous demandons si le texte Je t'offrirai une gazelle tournerait le dos au colonialisme, ou bien, au contraire, 
traduirait une réflexion propre, pour ne pas dire originale, du romancier, qui se manifeste par une écriture réflexive.

Afin de répondre à notre interrogation, nous émettons l'hypothèse suivante : la réflexion sur le fait colonial passerait par une réflexion sur soi ; en tant que sujet et écrivain - d'où la subjectivité de l'écriture. Pour étayer notre analyse, nous prendrons comme point d'appui les travaux de Lucien Dallenbach, exposés dans son ouvrage Le récit spéculaire (1977) sur la mise en abyme.

\section{Rappel historique}

Très présente dans le Nouveau roman, durant les années 1950 et 1960, la technique de mise en abyme remonte au début du $\mathrm{XX}^{\mathrm{e}}$ siècle avec André Gide. Le romancier français, dans son cahier - Journal publié en 1893 - a, pour la première fois, fait allusion à la technique de mise en abyme en écrivant : « J'aime assez qu'en une œuvre d'art on retrouve ainsi transposé, à l'échelle des personnages, le sujet même de cette œuvre » (Ibid. :15). Par cet aveu, l'auteur révèle sa préférence pour les histoires enchâssées. C'est-à-dire des récits traversés par ce que le théoricien Lucien Dallenbach appelle la mise en abyme. L'auteur du récit spéculaire définit, en s'appuyant sur le postulat gidien, ce procédé d'écriture : « est mise en abyme toute enclave entretenant une relation de similitude avec l'œuvre qui la contient » (1977: 18).

Cette acception s'applique à notre corpus, puisque ce dernier est composé de deux récits enchâssés : le premier est le projet littéraire, publication d'un roman ; le second est l'histoire d'amour, racontée dans le second texte, entre Yaminata, princesse bleue touareg du Sud (Sahara), et Moulay. 
Dans le même ouvrage, Le récit spéculaire. Essai sur la mise en abyme, $\mathrm{L}$. Dallenbach développe sa réflexion sur la mise en abyme, en notant : «[...] est mise en abyme tout miroir interne réfléchissant l'ensemble du récit par réduplication simple, répétée ou spécieuse » (1977 : 52). Ce miroir dont on parle ici prend forme dans le récit à travers l'histoire du romancier portant le projet d'édition de son manuscrit. L'écrivain raconte sa propre histoire. Notons, par ailleurs, que cette technique de mise en abyme donne au texte une forme de subjectivité que nous aborderons dans le point qui suit.

\section{Mise en abyme et subjectivité}

En effet, la mise en abyme est un procédé d'écriture qui pourrait être interprété comme une forme de subjectivité, qui permet au romancier de se démarquer de certains canons littéraires dits «classiques ». À travers le recours à cette technique narrative, l'auteur « s'auto-désigne » et pense son acte d'écrire. La subjectivité, comme pratique littéraire prégnante dans la production romanesque des écrivains occidentaux, pourrait paraitre comme une forme d'aliénation dans un récit appartenant à une aire culturelle où le «je » est, culturellement, banni. Ajoutons à cela, le contexte socio-historique dans lequel s'inscrit le récit, c'est-à-dire la période coloniale, où une bonne partie de romanciers algériens ont mis à nu, par leurs écrits littéraires, le colonialisme.

Dans le même ordre d'idée, écrire d'une manière poétique aux yeux de certains critiques mus par la fibre nationaliste - nous pensons ici à Mostefa Lacheraf que nous évoquerons dans le prochain point de la réflexion -, c'est tourner le dos aux siens. Dit autrement, l'écrivain, dans une telle situation, est censé se positionner en consacrant sa prose au service de la nation, prise sous le 
joug colonial, faisant ainsi de l'écriture un moyen de résistance contre l'oppresseur ; de ce fait, la dimension esthétique doit être reléguée au second plan. Or, dans Je t'offrirai une gazelle, le poétique semble primer sur le politique. Cette façon de construire la fiction dans le récit, et qui se base sur un procédé narratif que R. Barthes appelle la réflexivité (Barthes, 2002: 477), mérite d'être approfondi.

\section{Une écriture réflexive}

Selon Roland Barthes, la réflexivité consiste à parler de son propre langage : « c'est en quelques sorte le regard retourné du langage sur lui-même » (2002: 477). C'est la conscience orientée vers elle-même; de ce fait découle la subjectivité, laquelle est un procédé langagier qui résulte de la réflexivité dans le sens où le fait de parler de « soi » ne peut pas se faire d'une manière détachée ou objective.

Pour L. Dallenbach, tout « récit spéculaire », marqué par la technique de la mise en abyme, doit porter une «unité minimale » (Dallenbach, op. cit. : 5) appelée réflexivité. Celle-ci se manifeste dans Je t'offrirai une gazelle à travers des segments narratifs dans lesquels le romancier, Malek Haddad, parle d'un autre auteur ; qui n'est que lui-même. Afin d'illustrer notre argumentation, citons le segment narratif : l'écrivain écrit à la page 21, «L'AUTEUR est lourd. Il n'habite pas une maison bleue aux volets verts sur la colline » (Idem.).

Ici, le narrateur introduit l'auteur du second récit - le manuscrit remis à un éditeur - dans le roman. Nous avons d'autres passages où le romancier, déléguant la voix à son double, parle de lui-même, c'est-à-dire d'une manière réflexive : 
«l'auteur a eu peur. L'auteur sait qu'un destin c'est l'aboutissement des enchainements idiots. Une force aveugle ne dit pas sa puissance. Elle affirme son non-sens. L'auteur a eu peur et cette peur l'humilie » (op. cit. : 23). La répétition du vocable « auteur », à laquelle procède le narrateur, traverse tout le récit. Il y a plusieurs passages qui illustrent cette «obsession narrative ». Il en est ainsi, également, à titre d'exemple, à la page 46 : «Alors l'auteur veut parler de son livre. Ses yeux se redressent, ses doigts bavardent. C'est un poète, c'est un capitaine lorsqu'il parle de son livre. Il est aussi sérieux qu'un cordonnier » (Idem).

Nous pouvons parler ainsi de «métarécit» (Genette : 2004) dans la mesure où il s'agit de construction d'un récit dans un récit. Ainsi nous dirions que Je t'offrirai une gazelle est l'histoire constituant le sujet du roman que nous lisons. Passons à présent à un autre point de notre réflexion, qui est celui de la subjectivité en rapport avec le fait colonial.

\section{Subjectivité et contexte colonial}

À partir du texte, nous déduirons que l'auteur de Je t'offrirai une gazelle développe l'idée selon laquelle la prise de conscience, et par ricochet l'engagement dans un contexte marqué par la colonisation en faveur du pays dont il est originaire, passe, d'abord, par une réflexion sur soi en tant que sujet et écrivain. Dans le récit, M. Haddad réfléchit sur son statut de romancier-poète ; son rapport à l'écriture, à la langue et au monde de l'édition offre autant de thèmes, évoqués en filigrane et d'une manière poétique, illustrant la condition tragique. 
Cette façon de penser l'écriture a suscité une polémique auprès de la « critique littéraire » de l'époque, qui se veut moralisatrice et soucieuse des choix idéologiques des romanciers. Rappelons ainsi la critique dont a fait l'objet l'œuvre littéraire de M. Haddad par l'essayiste algérien, Mostefa Lacheraf :

Allons, il faut démystifier: Malek Haddad, Assia Djebar sont des écrivains qui n'ont jamais saisi nos problèmes, même les plus généraux. Ils ont tout ignoré, sinon de leur classe petite-bourgeoise, du moins de tout ce qui avait trait à la société algérienne ; de tous les écrivains algériens, ce sont eux qui connaissent le moins bien leur pays, ce qui les pousse à escamoter les réalités algériennes sous une croute "poétique", elle-même sans originalité du point de vue du roman : ribaude "chez l'un, bourgeoise chez l'autre". (Lacheraf, $1963: 733-734)$

Ce reproche fait à l'écrivain prouve que toute « aventure poétique », à l'époque (les années 50), était périlleuse. L'œuvre littéraire est contrainte, de par son contexte, à défendre la cause du peuple opprimé. Néanmoins, l'on peut remarquer que malgré la situation dans laquelle se trouve le pays dont sont issus les romanciers, c'est-à-dire le joug colonial, cela n'a pas empêché certains écrivains d'assumer les deux tâches : l'engagement politique et l'écriture - au sens d'esthétique - ; ce faisant, ils ont su répondre aux recommandations des dirigeants politiques du FLN, et ce, tout en restant des poètes. C'était le cas des auteurs comme K. Yacine et Malek Haddad.

Cela dit, ce qui retient notre attention dans le récit haddadien, à travers le rapport qu'entretient l'auteur avec la langue française, c'est la dimension tragique qui sous-tend le récit. 


\section{Esthétique de la transgression}

L'orthodoxie religieuse prédominant dans cette aire maghrébine, réglementée par la religion musulmane, à laquelle appartient M. Haddad, proscrit toute expression du «je » : «maudit soit le mot je / moi ». En partant de cette consigne cultuelle, nous sommes en droit de penser que le recours à l'écriture en employant le « je » au détriment d'un « nous » symbolisant la oumma, est une forme de transgression d'un ordre moral qui puise sa source dans le fait religieux. Par ailleurs, ne pas pouvoir écrire dans la langue maternelle et ne pas être à même de reproduire une parole «authentique », confèrent au texte une dimension tragique. Mais c'est aussi le fait de dire le monde subjectivement, et de ne pas être compris par les siens, qui inscrit le texte dans une tragédie. Celle-ci prend forme dans le récit à travers le rapport qu'entretient le romancier avec la langue de l'Autre et le langage d'une manière générale. En ce sens, le passage ci-après traduit le drame linguistique : «C'est un mot qui détonne au milieu des bouteilles. Avec cette pluie grasse et collante, ce ciel hypocrite, ces nuages de panique. C'est un mot orphelin. C'est un mot en exil. C'est un mot qui a froid. C'est un mot qui a mal au cœur » (Haddad, $1959: 46)$.

Cet extrait dans lequel le narrateur avoue son exil linguistique et son incapacité à faire parler la langue originelle, en empruntant un autre idiome que le sien, celui de l'Autre, est un signe de l'exil intérieur que vit un romancier condamné par les contraintes socio-historiques à s'exprimer dans des vocables « étrangers ». C'est ainsi que nous pourrions parler de déchirement linguistique du poète. 
En bref, ce qu'il faut retenir de la manière de concevoir l'écriture chez Malek Haddad, c'est bien la réflexion qu'il fait sur le langage. L'auteur, de par ses jeux d'écriture, montre qu'il est conscient du sens des mots et de leur poids. Ce procédé scriptural montre encore une fois que l'écrivain pense l'acte d'écrire. Nous pouvons considérer cette réflexivité comme une spécificité de l'écriture haddadienne, qui, à travers cette mise en abyme, s'inscrit dans la modernité.

De plus, cette façon de concevoir l'écriture par M. Haddad, où le poétique, plus exactement la subjectivité, traverse le récit, nous amène à réfléchir sur le rapport existant entre les trois concepts : subjectivité, art et engagement. Si l'on se réfère au philosophe français, Jean-Paul Sartre, on remarquerait que l'art, en particulier l'œuvre poétique, doit être au service de l'engagement; et que l'écrivain, en paraphrasant l'auteur de Le Diable et le Bon Dieu, est de toute façon, malgré lui, condamné à s'engager.

\section{Conclusion}

L'écriture, pour Malek Haddad, permet de faire parler le «je», sa subjectivité, et de témoigner sur la situation tragique de l'écrivain « francophone » dans un contexte marqué par la colonisation du pays d'origine. En donnant libre cours à sa "conscience malheureuse », le poète peint son intériorité. De fait, écrire, c'est d'abord penser le soi, c'est-à-dire sa propre condition de scripteur aux prises avec les vicissitudes de l'Histoire.

Ainsi, le procès que fait l'auteur à la colonisation se traduit par un récit poétique marqué par le seau de la subjectivité. Cette façon de concevoir l'acte d'écrire, qui a suscité des critiques de la part de ceux qui n'ont pas compris la 
profondeur de l'œuvre, montre que la réflexion sur le référent historique (le joug colonial) passe d'abord par une réflexion sur soi. De fait, la spécificité de l'écriture haddadienne consiste dans sa façon de penser la littérature, c'est-à-dire, eu égard du style imagé, une entreprise poétique, donc subjective. Et la subjectivité telle qu'elle prend forme dans le récit est avant tout une quête de l'amour, de soi et de la liberté.

En définitive, même si la création littéraire s'apparente, à travers les jeux d'écriture, à un « amusement », il n'en demeure pas moins que l'écrivain, de par le fait d'écrire dans une langue étrangère, ce qui lui rappelle son éloignement du pays d'origine et sa condition de colonisé, éprouve un malaise, voire un exil intérieur illustré par le procès du sujet fictif, représenté par le « je »; et symbolisé dans le récit par l'inaboutissement du projet littéraire - l'échec de la publication d'un roman - qui traduit cette crise du « scripteur ». 


\section{Bibliographie}

Amossy, Ruth, La présentation de soi. Ethos et identité verbale, Paris, Presses Universitaires de France, «L'Interrogation philosophique », 2010.

Barthes, Roland, Le degré zéro de l'écriture, Paris, Seuil, 1953.

Barthes, Roland, Euvres complètes, Paris, Seuil, vol. 3, 2002.

Dallenbach, Lucien, Le récit spéculaire. Essai sur la mise en abyme, Paris, Seuil, 1977.

Deleuze, Gilles ; Guattari, Félix, Capitalisme et schizophrénie, Tome 1 : 1'Antiœdipe, Paris, Éditions de Minuit, 1972.

Deleuze, Gilles, Empirisme et subjectivité, Paris, PUF, 2010.

Genette, Gérard, Figures III, Paris, Seuil, 1972.

Genette, Gérard, Métalepse. De la figure à la fiction, Paris, Seuil, 2004.

Haddad, Malek, Je t’offrirai une gazelle, Seuil, Paris, 1959.

Hamon, Philippe, «Texte littéraire et métalangage », Poétique, n 31, 1977, pp. 266-267.

Helkulla, Mervi : «Énonciation et subjectivité dans La Première Gorgée de bière et autres plaisirs minuscules », Poétique, $\mathrm{n}^{\mathrm{O}} 147,37^{\mathrm{e}}$ année, septembre 2006, pp. 317-325.

Jakobson, Roman, Essais de linguistique générale, Paris, Minuit, 1963-1973, 2 vol.

Lacheraf, Mostefa, «L'avenir de la culture algérienne », Les temps modernes, $\mathrm{n}^{\circ} 209,1963$, pp.733-734. 
Pier, John \& Schaeffer, Jean-Marie, Métalepses. Entorses au pacte de la représentation, Paris, École des Hautes Études en Sciences Sociales, 2005.

Sartre, Jean-Paul, Qu'est-ce que la littérature ? Gallimard, Paris, 1948. 